\title{
High-temperature oxide thermoelectrics
}

\author{
Ichiro Terasak** \\ Department of Physics, Nagoya University, Nagoya 464-8602, Japan
}

\begin{abstract}
We have evaluated the power factor of transition metal oxides at high temperatures using the Heikes formula and the Ioffe-Regel conductivity. The evaluated power factor is found to be nearly independent of carrier concentration in a wide range of doping, and well explains the experimental data for cobalt oxides. This suggests that the same power factor can be obtained with a thermopower larger than $2 k_{B} / e$, and also suggests a reasonably high value of the dimensionless figure of merit $Z T$. We propose an oxide thermoelectric power generator by using materials having a thermopower larger than $300 \mu \mathrm{V} / \mathrm{K}$.
\end{abstract}

PACS numbers: 72.20.Pa, 72.80.Ga, 71.27.+a

\section{INTRODUCTION}

Thermoelectrics is a technology that can convert heat into electricity and vice versa through the thermoelectric phenomena in semiconductors and metals $\underline{\underline{1}}$ Owing to the pressing needs for reduction of the carbon-dioxide emission, thermoelectric power generation has received a renewed interest from the viewpoint of waste-heat recovery into electric power. In particular, power generator at high temperature in air is highly desirable for waste heats from car engines and garbage furnaces. For such purposes, thermoelectric devices using oxide ceramics are quite promising, but the thermoelectric conversion efficiency is not satisfactory at present.

After the discovery of the good thermoelectric properties of the layered cobalt oxide $\mathrm{Na}_{x} \mathrm{CoO}_{2}, 2$ oxide thermoelectrics using transition-metal oxides is extensively investigated for the last decade. $\underline{\underline{3}-6}$ Compared with other thermoelectric materials, transition-metal oxides are quite unique in the sense that (i) the electron correlation is strong enough to make the Heikes formula valid for the thermopower above room temperature, $\underline{-9}$ (ii) the resistivity is barely metallic in which the mean free path is of the order of the unit cell length, and (iii) the spin fluctuation significantly affects the physical properties, 10,11 As a result, the thermoelectric parameters cannot be predicted from conventional semiconductor physics $\underline{1}$

Here we try to clarify how the power factor $S^{2} \sigma$ changes with carrier concentration and temperature in transition-metal oxides, where $S$ and $\sigma$ are the thermopower and the conductivity, respectively. Although the thermopower of many transition-metal oxides have been discussed with the Heikes formula,,$\frac{5,12}{12}$ there are only a few discussions on how the conductivity is described in the same conditions ${ }^{13}-16$ In particular, we wish to address the following two anomalies. First, an optimum carrier concentration $n_{0}$ that maximizes the power factor is of the order of $10^{21} \mathrm{~cm}^{-3}$, and depends strongly on temperature. Second, while the thermopower at $n_{0}$ often depends on materials, the maximized power factor is approximately $2-3 \mu \mathrm{W} / \mathrm{cmK}^{2}$, and is nearly independent of material species. These are seriously incompatible with the "collective wisdom" of thermoelectrics, where $n_{0}$ is of the order of $10^{19} \mathrm{~cm}^{-3}$, and the optimized thermopower is approximately $2 k_{B} / e$. To see these two anomalies we will take $\mathrm{La}_{1-x} \mathrm{Sr}_{x} \mathrm{CoO}_{3}$ as a typical example. The doped $\mathrm{LaCoO}_{3}$ has been extensively investigated as a possible candidate for thermoelectric oxide,,$^{17-20}$ and the thermoelectric parameters have been systematically measured in a wide range of carrier concentration. $21-23$ Iwasaki et al $\stackrel{23}{.}$ have measured the thermoelectric parameters using sixteen polycrystalline samples of $\mathrm{La}_{1-x} \mathrm{Sr}_{x} \mathrm{CoO}_{3}$ from $x=0$ to 0.40 in a wide range of temperature from 77 to $1100 \mathrm{~K}$. Their comprehensive study offers a good example to test the idea discussed in the present paper. We discuss a possible value of the figure of merit for such materials at high temperatures.

\section{THEORETICAL BACKGROUND}

Let us briefly summarize the conventional thermoelectrics by following Mahan's review article $\stackrel{1}{\underline{1}}$ According to the Boltzmann equation, the thermopower $S$ can be written with the Fermi-Dirac distribution $f_{0}$ and the chemical potential $\mu$ by

$$
S=\frac{1}{e T} \frac{\int\left(-\frac{\partial f_{0}}{\partial \varepsilon}\right)_{\varepsilon=\varepsilon_{\mathbf{k}}} v_{\mathbf{k}}^{2} \tau_{\mathbf{k}}\left(\varepsilon_{\mathbf{k}}-\mu\right) d^{3} k}{\int\left(-\frac{\partial f_{0}}{\partial \varepsilon}\right)_{\varepsilon=\varepsilon_{\mathbf{k}}} v_{\mathbf{k}}^{2} \tau_{\mathbf{k}} d^{3} k},
$$

where $\varepsilon_{\mathbf{k}}, v_{\mathbf{k}}$ and $\tau_{\mathbf{k}}$ are the energy, velocity and scattering time for an electron with a wavevector of $\mathbf{k}$, respectively. We can also express the carrier density $n$ as

$$
n=2 \int \frac{d^{3} k}{(2 \pi)^{3}} f_{0}\left(\varepsilon_{\mathbf{k}}\right) \text {. }
$$

Let us evaluate Eqs. (11) and (2) at high temperatures. The Fermi-Dirac distribution function $f_{0}$ can be approximated as the Maxwell-Boltzmann distribution as

$$
f_{0}=\frac{1}{\exp (x-\beta \mu)+1} \sim \exp (\beta \mu-x),
$$

where $\beta=1 / k_{B} T$ and $x=\varepsilon_{\mathbf{k}} / k_{B} T$. Now we can evaluate the integrals in Eqs. (1) and (2), by assuming a single 
band with a parabolic dispersion. Then we get $n$ as

$$
n=\frac{1}{\pi^{2}}\left(\frac{2 m k_{B} T}{\hbar^{2}}\right)^{3 / 2} \frac{\sqrt{\pi}}{2} \exp (\beta \mu)
$$

For many practical cases, this equation determines the chemical potential $\mu$ as a function of $n$ and $T$ as

$$
\mu=k_{B} T \ln \left(\frac{n}{\xi}\right)
$$

where

$$
\xi \equiv \frac{1}{\pi^{2}} \frac{\sqrt{\pi}}{2}\left(\frac{2 m k_{B} T}{\hbar^{2}}\right)^{3 / 2}
$$

In the same way, we evaluate the thermopower as

$$
\begin{aligned}
S & =-\frac{k_{B}}{e} \frac{\int d^{3} k v_{\mathbf{k}}^{2} \tau_{\mathbf{k}}(\beta \mu-x) \exp (\beta \mu-x)}{\int d^{3} k v_{\mathbf{k}}^{2} \tau_{\mathbf{k}} \exp (\beta \mu-x)} \\
& =-\frac{k_{B}}{e}(\beta \mu-\langle x\rangle), \\
& =-\frac{k_{B}}{e}\left(\ln \left(\frac{n}{\xi}\right)-\langle x\rangle\right)
\end{aligned}
$$

where $\langle x\rangle$ is the average of $x$ with a weight function of $v_{k}^{2} \tau_{\mathbf{k}} \exp (\beta \mu-x)$. Note that we used Eq. (5) to eliminate $\mu$. Now we can get the optimum condition of the power factor by differentiating it with $n$. By neglecting the carrier-concentration dependence of the mobility, we get

$$
\frac{d\left(S^{2} \sigma\right)}{d n} \propto \frac{d}{d n}\left[n(\ln (n / \xi)-\langle x\rangle)^{2}\right]=0
$$

Then the optimum carrier concentration $n_{0}$ should satisfy

$$
\ln \left(\frac{n_{0}}{\xi}\right)=\langle x\rangle-2
$$

By eliminating $\langle x\rangle$ from Eq. (77), we get the optimum thermopower $S_{0}$ as

$$
S_{0}=\frac{k_{B}}{e}\left(-\ln \left(\frac{n_{0}}{\xi}\right)+\langle x\rangle\right)=\frac{2 k_{B}}{e}
$$

This indicates that the optimum thermopower is universal and independent of materials parameters. Using realistic parameters for $x$, we can evaluate $n_{0}$ to be of the order of $10^{19} \mathrm{~cm}^{-3}$ at $300 \mathrm{~K}$. In fact, the conventional thermoelectric materials are optimized around this carrier concentration, and show a thermopower of \pm 200 $\mu \mathrm{V} / \mathrm{K}$ that is close to $\pm 2 k_{B} / e= \pm 170 \mu \mathrm{V} / \mathrm{K}$. Very recently, Pichanusakorn and Bandaru ${ }^{24}$ have performed a similar but more extensive calculation, and have found that the optimized thermopower is always in a limited range of $130-187 \mu \mathrm{V} / \mathrm{K}$, which is essentially independent of material parameters.

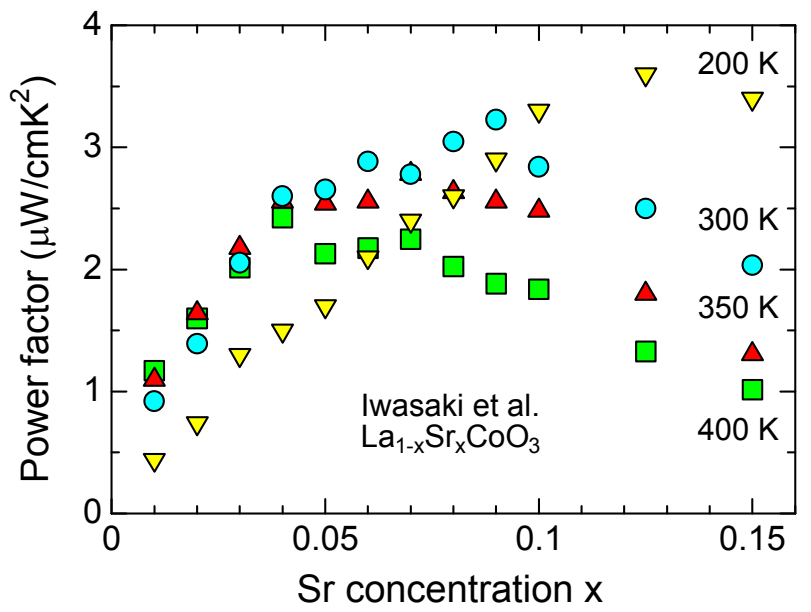

FIG. 1. (Color online) The power factor of polycrystalline samples of $\mathrm{La}_{1-x} \mathrm{Sr}_{x} \mathrm{CoO}_{3}$ measured by Iwasaki et al ${ }^{23}$ plotted as a function of Sr concentration $x$.

\section{POWER FACTOR FOR DISORDERED SYSTEMS}

According to Eq. (9), $n_{0}$ is proportional to $T^{3 / 2} \exp (\langle x\rangle-2)$, meaning that the optimum carrier concentration is higher for higher temperatures. This is not true in real transition-metal oxides. Figure 1 shows the power factor of polycrystalline samples of $\mathrm{La}_{1-x} \mathrm{Sr}_{x} \mathrm{CoO}_{3}$ measured by Iwasaki et al ${ }^{23}$ as a function of $\mathrm{Sr}$ concentration $x$ for several temperatures. The optimum $\mathrm{Sr}$ concentration $x_{0}$ depends clearly on temperature. This is reasonable, because Eq. (8) is based on the assumption that the mobility is independent of carrier concentration. In real materials, however, the mobility is highly dependent on carrier concentration especially near metalinsulator transitions.

Let us have a closer look at the temperature dependence of the optimum Sr concentration $x_{0}$. While $x_{0}$ is around 0.12 (corresponding to $n=2 \times 10^{21} \mathrm{~cm}^{-3}$ ) at 200 $\mathrm{K}, x_{0}$ shifts to lower $x$ with increasing temperature. The power factor at $350 \mathrm{~K}$ shows a plateau from $x=0.04$ to 0.1 , which is understood from two broad maxima located near $x=0.04$ and 0.1 . In this context, $x_{0}$ gradually decreases with temperature from $x_{0}=0.12$ at $200 \mathrm{~K}$, through 0.1 at $300 \mathrm{~K}$, to 0.04 at $400 \mathrm{~K}$.

Let us discuss an origin for the temperature dependence of $x_{0}$. In $\mathrm{La}_{1-x} \mathrm{Sr}_{x} \mathrm{CoO}_{3}$, the thermopower from 200 to $400 \mathrm{~K}$ are weakly dependent on temperature. As is widely known, the temperature-independent thermopower has been analyzed using the Heikes formula? In this formula, entropy per site is associated with the thermopower. In a simplest case, the thermopower of the Heikes formula $S_{\mathrm{H}}$ can be given by

$$
S_{\mathrm{H}}=\frac{k_{B}}{e} \ln \frac{2 x}{1-x},
$$

where $x$ is the carrier concentration per unit cell. This 


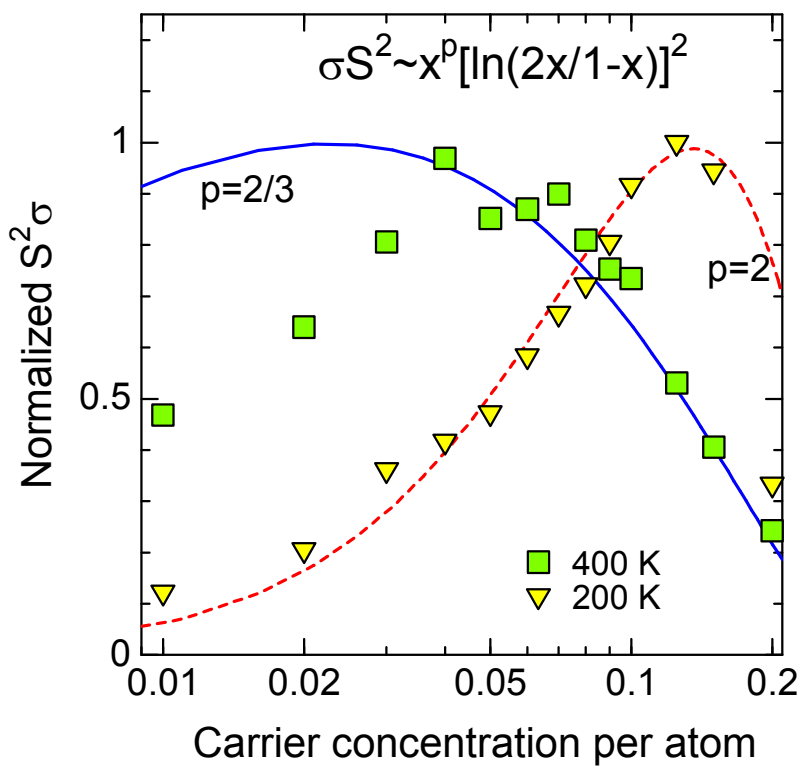

FIG. 2. (Color online) The power factor calculated from Eq. (13) (solid curve) and Eq. (14) (dotted curve). plotted as a function of carrier concentration per unit cell. The power factors at 200 and $400 \mathrm{~K}$ in Fig. 1 are also plotted.

formula is valid, when the thermal energy $k_{B} T$ is much larger than the transfer energy $t$ but much smaller than the on-site Coulomb repulsion $U\left(t \ll k_{B} T \ll U\right)$. Although the spin and orbital degrees of freedom exist in the Co ions, we neglect in the present paper to avoid the controversy of the spin states of the doped $\mathrm{LaCoO}_{3}, \underline{22,25}, \underline{26}$

In the same condition, we should employ the conductivity for nonmetallic conduction. One extreme case is known as the Ioffe-Regel limit, where the electron mean free path is close to the lattice parameters. In this picture, the Ioffe-Regel conductivity $\sigma_{\mathrm{IR}}$ is given by

$$
\sigma_{\mathrm{IR}}=0.33 x^{2 / 3} \frac{e^{2}}{\hbar a},
$$

where $a$ is the lattice parameter $\stackrel{27}{=}$ Thus the power factor is written as

$$
S_{\mathrm{H}}^{2} \sigma_{\mathrm{IR}} \propto x^{2 / 3}\left[\ln \frac{2 x}{1-x}\right]^{2} .
$$

Another extreme case is the electrical conduction near the Anderson transition. In this case, the conductivity $\sigma_{\mathrm{A}}$ goes toward zero critically as $\sigma_{\mathrm{A}} \propto\left(n-n_{c}\right)^{p}$, when $n$ approaches the critical concentration $n_{c}$. When the critical regime is sufficiently large, we get $\sigma_{\mathrm{A}} \propto n^{p}$ for $n \gg n_{c}$. In this case, the power factor is written as

$$
S_{\mathrm{H}}^{2} \sigma_{\mathrm{A}} \propto x^{p}\left[\ln \frac{2 x}{1-x}\right]^{2} .
$$

Figure 2 shows the normalized power factors calculated from Eqs. (13) and (14), together with the experimental data shown in Fig.1, where the properly normalized values of $x^{p}[\ln 2 x /(1-x)]^{2}$ is plotted for $p=2 / 3$ [Eq. (13)] and for $p=2$ [Eq. (14)] by the solid and dotted curves, respectively. As is clearly seen by the dotted curve in Fig. 2, the exponent of $p=2$ well explains the power factor measured at $200 \mathrm{~K}$. This suggests that the mobility is proportional to $x$, which is consistent with the mobility at $300 \mathrm{~K}$ evaluated by Iwasaki et al. $\stackrel{23}{ }$ This doping dependent mobility is the reason why the optimum carrier concentration is much larger than the value given by Eq. (91). At higher temperature, in contrast, all the samples tend to show $\sigma_{\mathrm{IR}}$ called resistivity saturation, 27 and the solid curve explains the measured data at $400 \mathrm{~K}$ for $x>0.03$. It is reasonable that the measured data is below the solid curve for $x \leq 0.03$, because at such low carrier concentration, strong localization takes place to make the conductivity lower than $\sigma_{\mathrm{IR}}$.

Looking at Eqs. (13) and (14), one can understand why the thermoelectric performance of the thermoelectric oxides is poor at room temperature, but is comparable to that of other materials above $1000 \mathrm{~K}$. The low-temperature mobility is poorer for lower carrier concentration, and the conductivity $\sigma_{\mathrm{A}}$ is much lower than that of the conventional thermoelectric materials. On the other hand, when Eq. (13) is valid, the resistivity and thermopower become independent of temperature. For $x=0.02$ and $a=5 \AA$, for example, we have $\sigma_{\mathrm{IR}}=100$ $\mathrm{S} / \mathrm{cm}$ and $S_{\mathrm{H}}=300 \mu \mathrm{V} / \mathrm{K}$. Then the power factor equals $9 \mu \mathrm{W} / \mathrm{cmK}^{2}$. Note that the value of $9 \mu \mathrm{W} / \mathrm{cmK}^{2}$ is the upper limit for materials in which the Heikes formula is valid. It is thus reasonable to regard this value as close to the observed maximum power factor of $2-3 \mu \mathrm{W} / \mathrm{cmK}^{2}$ in Fig. 1. Assuming a low thermal conductivity $\kappa$ of 10 $\mathrm{mW} / \mathrm{cmK}$, we estimate the figure of merit $Z=S_{\mathrm{H}}^{2} \sigma_{\mathrm{IR}} / \kappa$ to be $9 \times 10^{-4} \mathrm{~K}^{-1}$, which gives $Z T=0.9$ at $1000 \mathrm{~K}$. The thermoelectric parameters of the layered cobalt oxides roughly meet the above conditions $\underline{28-33}$ A prime example is $\mathrm{Ca}_{3} \mathrm{Co}_{4} \mathrm{O}_{9}$; At $1000 \mathrm{~K}$, a single crystal shows a power factor of $26 \mu \mathrm{W} / \mathrm{cmK}^{2}$ with a resistivity of 2.4 $\mathrm{m} \Omega \mathrm{cm}$ and a thermopower of $250 \mu \mathrm{V} / \mathrm{K}, 31$ and a ceramic sample shows a power factor of $6 \mu \mathrm{W} / \mathrm{cmK}^{2}$ with a resistivity of $9 \mathrm{~m} \Omega \mathrm{cm}$ and a thermopower of $230 \mu \mathrm{V} / \mathrm{K} \stackrel{33}{\underline{3}}$ We should further emphasize that the above estimation is done in the limit of low mobility. This is also incompatible against the collective wisdom of thermoelectrics, where high-mobility semiconductors are required for high $Z T$ values. Equation (13) warrants that highly disordered materials can be fairly good thermoelectric materials at high temperature.

Another feature of Eq. (13) is that the solid curve in Fig. 2 indicates that the power factor has a broad maximum around $0.01-0.06$. This is because $\sigma_{\mathrm{IR}}$ is weakly dependent on $x$ so that the increase in $S_{\mathrm{H}}$ compensates the decrease in $\sigma_{\mathrm{IR}}$ well. Equation (11) gives 340 and $170 \mu \mathrm{V} / \mathrm{K}$ for $x=0.01$ and 0.06 , respectively, and materials with a thermopower larger than $300 \mu \mathrm{V} / \mathrm{K}$ should be designed to maximize the power factor. In fact, the thermopower for $x=0.03$ is $300 \mu \mathrm{V} / \mathrm{K}$ at $400 \mathrm{~K}$. 
This is again seriously incompatible with the prediction of Eq. (10) that the optimized thermopower is always $2 k_{B} / e=170 \mu \mathrm{V} / \mathrm{K}$.

Kobayashi et al ${ }^{34}$ have reported that the power factor of the misfit layered cobalt oxides $\left[\mathrm{Bi}_{2} \mathrm{~A}_{2} \mathrm{O}_{4}\right]\left[\mathrm{CoO}_{2}\right]_{b_{1} / b_{2}}$ is nearly independent of materials at $300 \mathrm{~K}$. By changing the block layer, they controlled the carrier concentration, and concomitantly changed the resistivity and thermopower from sample to sample. An important finding is that the power factor at $300 \mathrm{~K}$ is close to $2 \mu \mathrm{W} / \mathrm{cmK}^{2}$ for all the samples measured. We think this dopingindependent power factor is essentially understood with Eq. (13).

The present work suggests that the thermopower of transition-metal oxides can be increased up to $340 \mu \mathrm{V} / \mathrm{K}$ with remaining the power factor unchanged, when the transport parameters are explained with the Heikes formula. By using such oxides, one can reduce the number of thermocouple without deteriorating the performance of the device. Such devices are easier to fabricate, easier to lower contact resistance, and easier to take impedance matching because of its high impedance. We hope that we can increase the yielding ratio and the reliability as well by using such devices. Recently, Bonetti et al 35 have reported that a huge thermopower of $7 \mathrm{mV} / \mathrm{K}$ around 30 $45^{\circ} \mathrm{C}$ for some electrolytes, and argued the same idea.

\section{SUMMARY}

We have discussed the high-temperature power factor of disordered materials using the Heikes formula for the thermopower and the Ioffe-Regel limit for the conductivity. We have found that this power factor can be as large as $9 \mu \mathrm{W} / \mathrm{cmK}^{2}$ (a resistivity of $10 \mathrm{~m} \Omega \mathrm{cm}$ and a thermopower of $300 \mu \mathrm{V} / \mathrm{K})$ at any temperatures, which could give a reasonable value of $Z T=0.9$ at $1000 \mathrm{~K}$ with a realistic value of the thermal conductivity of 10 $\mathrm{mW} / \mathrm{cmK}$. We have further found that this power factor takes a broad maximum for a wide range of carrier concentration from 0.01 to 0.06 per unit cell. Since this carrier concentration range corresponds to a thermopower from 170 to $340 \mu \mathrm{V} / \mathrm{K}$, a thermopower larger than 300 $\mu \mathrm{V} / \mathrm{K}$ is applicable for a thermoelectric power generator using transition-metal oxides.

\section{ACKNOWLEDGMENTS}

The author wish to thank Y. Horiuchi, D. Sawaki, A. Inagoya for collaboration on experimental searches for large-thermopower materials, and also appreiate W. Kobayashi and S. Hébert for stimulating input about the doping-independent power factor. This work was partially supported by Tokuyama Science Foundation, and by Strategic Japanese-Finland Cooperative Program on "Functional Materials", JST, Japan.
* Email me at terra@cc.nagoya-u.ac.jp

${ }^{1}$ G. M. Mahan, Solid State Physics 51, 81 (1998).

2 I. Terasaki, Y. Sasago, and K. Uchinokura, Phys. Rev. B 56, R12685 (1997).

3 I. Terasaki, Physica B 328, 63 (2003).

4 A. Maignan, L. B. Wang, S. Hébert, D. Pelloquin, and B. Raveau, Chem. Mater. 14, 1231 (2002).

5 A. Maignan, S. Hébert, L. Pi, D. Pelloquin, C. Martin, C. Michel, M. Hervieu, and B. Raveau, Crystal Engineering 5, 365 (2002).

${ }^{6}$ K. Koumoto, I. Terasaki, and R. Funahashi, MRS Bulletin 31, 206 (2006).

7 P. M. Chaikin and G. Beni, Phys. Rev. B 13, 647 (1976).

8 T. T. M. Palstra, A. P. Ramirez, S. W. Cheong, B. R. Z. and, P. Schiffer, and J. Zaanen, Phys. Rev. B 56, 5104 (1997).

9 W. Koshibae, K. Tsutsui, and S. Maekawa, Phys. Rev. B 62, 6869 (2000).

10 P. Limelette, S. Hébert, V. Hardy, R. Frésard, C. Simon, and A. Maignan, Phys. Rev. Lett. 97, 046601 (2006).

11 J. Bobroff, S. Hébert, G. Lang, P. Mendels, D. Pelloquin, and A. Maignan, Phys. Rev. B 76, 100407 (2007).

12 S. Hébert, D. Flahaut, C. Martin, S. Lemonnier, J. Noudem, C. Goupil, A. Maignan, and J. Hejtmanek, Prog.
Solid State Chem. 35, 457 (2007).

13 A. Kapitulnik, Appl. Phys. Lett. 60, 180 (1992).

14 G. Pálsson and G. Kotliar, Phys. Rev. Lett. 80, 4775 (1998).

15 K. Durczewski and A. Krzywicki, Phys. Rev. B 58, 10302 (1998).

16 W. Koshibae and S. Maekawa, Phys. Rev. Lett. 87, 236603 (2001).

17 P. Migiakis, J. Androulakis, and J. Giapintzakis, J. Appl. Phys. 94, 7616 (2003).

18 J. Androulakis, P. Migiakis, and J. Giapintzakis, Appl. Phys. Lett. 84, 1099 (2004).

19 R. Robert, M. Aguirre, P. Hug, A. Reller, and A. Weidenkaff, Acta Materialia 55, 4965 (2007).

20 Z. Jirák, J. Hejtmánek, K. Knívzek, and M. Veverka, Phys. Rev. B 78, 014432 (2008).

21 H. Masuda, T. Fujita, T. Miyashita, M. Soda, Y. Yasui, Y. Kobayashi, and M. Sato, J. Phys. Soc. Jpn. 72, 873 (2003).

${ }^{22}$ M. Kriener, C. Zobel, A. Reichl, J. Baier, M. Cwik, K. Berggold, H. Kierspel, O. Zabara, A. Freimuth, and T. Lorenz, Phys. Rev. B 69, 094417 (2004).

${ }^{23}$ K. Iwasaki, T. Ito, T. Nagasaki, Y. Arita, M. Yoshino, and T. Matsui, J. Solid State Chem. 181, 3145 (2008). 
${ }^{24}$ P. Pichanusakorn and P. R. Bandaru, Appl. Phys. Lett. 94, 223108 (2009).

${ }^{25}$ K. Asai, O. Yokokura, N. Nishimori, H. Chou, J. M. Tranquada, G. Shirane, S. Higuchi, Y. Okajima, and K. Kohn, Phys. Rev. B 50, 3025 (1994).

26 J. Wu and C. Leighton, Phys. Rev. B 67, 174408 (2003).

27 M. Gurvitch, Phys. Rev. B 24, 7404 (1981).

28 M. Ohtaki, Y. Nojiri, and E. Maeda, in Proceedings of The 19th International Conference on Thermoelectrics (ICT2000), edited by M. Rowe (Babrow, Cardiff, 2000) pp. 190-195.

29 K. Fujita, T. Mochida, and K. Nakamura, Jpn. J. Appl. Phys. Part 1 40, 4644 (2001).
30 R. Funahashi and M. Shikano, Appl. Phys. Lett. 81, 1459 (2002).

31 M. Shikano and R. Funahashi, Appl. Phys. Lett. 82, 1851 (2003)

32 M. Ito, T. Nagira, D. Furumoto, S. Katsuyama, and H. Nagai, Scripta Materialia 48, 403 (2003).

33 N. Van Nong, N. Pryds, S. Linderoth, and M. Ohtaki, Advanced Materials 23, 2484 (2011).

34 W. Kobayashi, S. Hébert, H. Muguerra, D. Grebille, D. Pelloquin, and A. Maignan, in Proceedings of the 26th International Conference on Thermoelectrics (ICT2007) (IEEE, Piscataway, 1997) pp. 117-120.

35 M. Bonetti, S. Nakamae, M. Roger, and P. Guenoun, arXiv.org (J. Chem. Phys. submitted), 1102.2425(2011). 